Therefore, we introduce the anterior approach as radical operation of this involvement. Making the thoracotomy, resection of involved disc followed by bone graft is performed under direct vision.

This method has been practiced in two cases and their results were better, although their postoperative periods were short. Degenerative changes observed in the resected specimen proves the rationality of this performance.

\title{
120. Effect of Anterior Cervical Fusion on the Clinical Manifestations of Cervical Disorders, especially on the Symptoms on the Trunk and Disfunction of the Bladder and the Colon
}

\author{
Yutaka OnJI, Kisei UEda and Yomei IwASAKI \\ Department of Orthopedic Surgery, Nara Medical College and Otemae Hospital, Osaka
}

Clinical characteristics of cervical disorders including cervical spondylosis and trauma on the cervical spine, are very complex. The disease attracted much attension in recent years. As a result, many studies have been done on the symptoms and signs due to the cervical disorders. However, little is known about the symptoms developed on the trunk and intestine due to the cervical disorders. The present authors are going to discuss on chest pain, back pain, low back pain, dullness on the low back, pulling pain from the buttock to the thigh and tightness around the chest and the abdomen in relation with the cervical disorders. Disfunctions of bladder and bowel are also produced by the cervical disorders. Seventy patients including 31 cervical spondylosis, 19 cervical trauma and 20 cervical spodylosis accompanied by trauma, have been operated on by anterior interbody fusion. The shortest follow-up period is 6 months. Among the patients, 16 cases showed symptoms on the trunk and 15 showed disfunctions of the bladder and bowel. 